\title{
ChIP-seq Analysis to Explore DNA Replication Profile in Trifluridine-treated Human Colorectal Cancer Cells In Vitro
}

\author{
TAKASHI KOBUNAI ${ }^{*}$, KAZUAKI MATSUOKA $^{2 *}$ and TEIJI TAKECHI ${ }^{1}$ \\ ${ }^{I}$ Taiho Pharmaceutical Co., Ltd., Translational Research Laboratory, Tokyo, Japan; \\ ${ }^{2}$ Taiho Pharmaceutical Co., Ltd., Translational Research Laboratory (Tokushima office), Tokushima, Japan
}

\begin{abstract}
Background/Aim: Trifluridine (FTD) is a key component of the novel oral antitumor drug trifluridinel tipiracil that has been approved for the treatment of metastatic colorectal cancer. In this study, a comprehensive analysis of DNA replication profile in FTD-treated colon cancer cells was performed. Materials and Methods: HCT-116 cells were exposed to BrdU or FTD and subjected to DNA immunoprecipitation. Immunoprecipitated DNA was sequenced; the density of aligned reads along the genome was calculated. Peak finding, gene ontology, and motif analysis were performed using MACS, GREAT, and MEME, respectively. Results: We identified 6,043 and 5,080 high-confidence FTD and BrdU peaks in HCT116 cells, respectively. Of 6,043 FTD peaks, 2,911 peaks were uncommon to BrdU. We observed that FTD was preferentially incorporated into genomic regions containing simple repeats, $C p G$ islands, and gene bodies. Conserved motifs in FTD peaks contained dinucleotide repeats such as (GT)n. Conclusion: Global FTD incorporation patterns delineated FTD, preferentially incorporating loci in cancer cells.
\end{abstract}

Trifluridine/tipiracil (FTD/TPI), also known as TAS-102, is approved for the treatment of patients with metastatic colorectal cancer and advanced gastric cancer. A phase III clinical study in patients with metastatic colorectal cancer (CRC) refractory to standard chemotherapies showed that FTD/TPI significantly improved overall and progression-free survival compared to those of the placebo group (1). Moreover, this combination of drugs showed an encouraging

This article is freely accessible online.

*These Authors contributed equally to this study.

Correspondence to: Takashi Kobunai, Ph.D., Taiho Pharmaceutical Co., Ltd., Translational Research Laboratory, 1-2-4 Uchikanda, Chiyoda-ku, Tokyo 101-0047, Japan. Tel.: +81 332935394, Fax: +81 332934798, e-mail: takashi_kobunai@taiho.co.jp

Key Words: Trifluridine, trifluridine/tipiracil (TAS-102), ChIP-seq, colorectal cancer. efficacy and a tolerable safety profile for previously treated metastatic colorectal cancer (2).

In basic research, FTD, the active antitumor component of FTD/TPI, is an antineoplastic thymidine analog (3), efficiently incorporated into genomic DNA in tumor cells (4-7). FTD transported into the cytoplasm of tumor cells by equilibrative nucleoside transporter 1 and 2 is phosphorylated to monophosphate, diphosphate, and triphosphate (FTD-TP) forms by thymidine kinase 1 and nucleoside diphosphate kinase, respectively. DNA polymerase $\alpha$ incorporates FTD-TP into DNA at sites aligned with adenine on the opposite strand (8). FTD/TPI exerts its antitumor activity in vivo predominantly due to its DNA incorporation (7), rather than as a result of thymidilate synthase inhibition (6). However, the detailed molecular mechanisms underlying the cytotoxic effects after FTD incorporation into DNA remain uncertain.

An approach that combines 5-bromo-2'-deoxyuridine (BrdU)-labeled DNA immunoprecipitation with DNA microarray or next generation sequencing (ChIP-seq) to enable the genome-wide identification of BrdU-labeled chromosomal DNA has been used to study DNA replication, DNA repair, and DNA metabolism $(9,10)$. Recently, Kitao et al. reported that several antibodies against $\mathrm{BrdU}$ specifically cross-react with FTD incorporated into DNA (11). Therefore, we used ChIP-seq analysis to assess DNA replication profiles in FTD treated cells in order to explore the mode of FTD incorporation into genomic DNA in comparison with $\mathrm{BrdU}$, which is a non-cytotoxic compound.

\section{Materials and Methods}

Chemicals and antibody. FTD, BrdU, and Anti-BrdU antibody 3D4 (Cat\#555627) were purchased from Yuki Gosei Kogyo (Tokyo, Japan), Tokyo Chemical Industry (Tokyo, Japan), and BD Biosciences (San Jose, CA, USA), respectively.

Cell lines. The human colorectal cancer cell line HCT-116 was obtained from the American Type Culture Collection (ATCC, Manassas, VA, USA). Cells were cultured at $37^{\circ} \mathrm{C}$ in a humidified atmosphere with $5 \% \mathrm{CO}_{2}$ in Dulbecco's modified Eagle medium (Sigma-Aldrich, St. Louis, MO, USA) supplemented with $10 \%$ fetal 
bovine serum (Sigma-Aldrich), $100 \mathrm{U} / \mathrm{ml}$ penicillin (Nacalai Tesque, Kyoto, Japan), and $100 \mathrm{mg} / \mathrm{ml}$ streptomycin (Nakcalai Tesque). These cells were authenticated in 2014 by analyzing short tandem repeats.

ChIP-sequencing and data analyses. We obtained the DNA replication profile of HCT-116 cells as follows: asynchronous cultures of HCT-116 were exposed to $5 \mu \mathrm{M}$ BrdU, a non-cytotoxic control drug, or FTD for $4 \mathrm{~h}$. The cells $\left(4 \times 10^{7}\right.$ cells per sample) were then harvested, and subjected to DNA immunoprecipitation using an anti-BrdU antibody as described previously (12). Briefly, cell lysates were sonicated and the DNA was sheared to an average length of 300-500 bp. Genomic DNA (Input) was prepared by treating aliquots of chromatin with RNase, and proteinase $\mathrm{K}$, followed by ethanol precipitation. An aliquot of chromatin $(25 \mu \mathrm{g})$ was pre-cleared with protein A agarose beads (Invitrogen, Carlsbad, CA, USA). Genomic DNA regions of interest were isolated using 3 $\mu l$ of anti-BrdU antibody. Complexes were washed, eluted from the beads with SDS buffer, and subjected to RNase and proteinase K treatment. ChIP DNA was purified by phenol-chloroform extraction and ethanol precipitation. Illumina sequencing libraries were prepared from the ChIP and Input DNAs by the standard consecutive enzymatic steps of end-polishing, dA-addition, and adaptor ligation. After a final PCR amplification step, the resulting DNA libraries were quantified and sequenced on Illumina's NextSeq 500 (Illumina, San Diego, CA, USA). Sequence analysis was performed as follows: The 75-nt length sequence reads were mapped to the human genome (hg19) using the BWA algorithm (13). Only reads that passed Illumina's purity filter, aligned with no more than two mismatches, and mapped uniquely to the genome were used in the subsequent analysis. Also, duplicate reads were removed from the analysis. Determination of Fragment Density was performed as follows: To identify the density of fragments, the genome was divided into 32-nt bins and the number of tags and, 5 '-ends of the aligned reads in each bin was determined. Peak finding was performed using the MACS algorithm, which identifies significant enrichments in the ChIP-seq data file when compared to the Input data file ( random background). To compare peak metrics between two samples, overlapping intervals are grouped into "active regions," which are defined by the start coordinate of the most upstream interval and the end coordinate of the most downstream interval (=union of overlapping intervals; "merged peaks"). In locations where only one sample has an interval, this interval defines the active region. A comparison of active regions along genomic coordinates was performed using Strand NGS software ver.3.2 (Strand Life Science. Bangalore, India). The 60-bp sequences surrounding the summits of the top 1000 peaks were analyzed for conserved motifs using the online program MEME Suite ver. 5.0.4 (14). Gene ontology analysis was performed using Genomic Regions Enrichment of Annotations Tool (GREAT) (15).

Statistical analysis. Fisher's exact test and Spearman's rank correlation evaluation were performed with JMP ver.13 (SAS Institute Inc., Cary, NC, USA). Results were considered statistically significant if $p<0.05$. Motifs with E-value $<0.05$ (MEME output) were considered significant.

\section{Results}

The numbers of active regions. We observed significant ChIP peaks over input DNA and identified 6,043 and 5,080 high confidence FTD and BrdU active regions, respectively, in HCT-116 cells (Table I). Interestingly, 2,911 out of 6,043 FTD active regions were uncommon to those of BrdU, while the remaining 3,132 FTD active regions were common to BrdU. The ChIP fragment densities along the genome are shown in Figure 1. The examples of FTD-specific ChIP peaks and common ChIP peaks between FTD and BrdU are shown in Figure 2. The number of FTD ChIP peaks were significantly correlated to the number of $\mathrm{CpG}$ islands (Spearman's Rho $\mathrm{r}=0.77, p<0.001$ ), but not to chromosomal size $(\mathrm{r}=0.28, p=0.19)$ and the number of simple repeats $(\mathrm{r}=0.33, p=0.12)$. Besides, the number of BrdU ChIP peaks was significantly correlated to the number of $\mathrm{CpG}$ islands ( $\mathrm{r}=0.67, p=0.0004)$, the number of simple repeats $(\mathrm{r}=0.50$, $p=0.013)$, and chromosomal size $(\mathrm{r}=0.46, p=0.025)$. These results suggested that the FTD peaks were not randomly distributed throughout the genome and some sequence preferences exist during FTD incorporation into DNA strand.

ChIP peak distributions. The median length of FTD or BrdU active regions was $500 \mathrm{bp}$ or $501 \mathrm{bp}$, respectively. Therefore, we generated two sets of 50,000 random control regions of 500-bp length over the entire hg19 genome as a control to compare with those of FTD or BrdU peaks. We analyzed FTD or BrdU active regions across each target region. Overlapping percentages of the active regions with genomic annotations are presented in Table II. Overlapping between FTD active regions and genomic regions such as simple repeat $(p<0.05)$, gene and gene prediction (including coding exon and intron) $(p<0.05)$ were significantly higher than those of BrdU active regions. When compared to the random control, the overlapping percentage of FTD active regions was 11 times higher for simple repeat $(p<0.0001), 3.6$ times higher for the replication origin $(p<0.0001)$, and 2.5 times higher for the CpG islands $(p<0.0001)$, respectively. Simple repeats in hg19 genome consist of $6.6 \%$ of mono-nucleotide repeat, $47 \%$ of di-nucleotide repeats, $10.5 \%$ of tri-nucleotide repeats, $20.6 \%$ of tetra-nucleotide repeats, $9.5 \%$ of penta-nucleotide repeats, and $5.8 \%$ of hexa-nucleotide repeats. FTD active regions overlapped mono-nucleotide repeats by $0.02 \%$, di-nucleotide repeats by $45.2 \%$, tri-nucleotide repeats by $9.1 \%$, and tetranucleotide repeats by $22.3 \%$, respectively (Table III). These findings also supported that the distribution of FTD peaks along the genome showed specific sequence preferences.

The association of FTD-ChIP intervals to the TSS of genes estimated by GREAT. ChIP-peaks were distributed across an entire genome including non-coding regions lacking annotation such as their biological functions. The GREAT is useful in studying cis functions of sets of non-coding genomic regions (15). Therefore, we applied GREAT on ChIP-seq data to identify the genes associated with distant peaks of FTD. FTD peaks occurred near genes involved in 
Table I. Summary of chromatin immunoprecipitation-DNA sequencing (ChIP-seq) active regions detected along the chromosomes.

\begin{tabular}{|c|c|c|c|c|c|}
\hline Chromosome & \# FTD active regions & \# BrdU active regions & Length (bp) ${ }^{1}$ & \# CpG Islands ${ }^{1}$ & \# Simple repeat $^{1}$ \\
\hline 1 & 454 & 343 & $249,250,621$ & 2,462 & 31,509 \\
\hline 2 & 407 & 430 & $243,199,373$ & 1,688 & 33,890 \\
\hline 3 & 176 & 132 & $198,022,430$ & 1,163 & 26,439 \\
\hline 4 & 223 & 194 & $191,154,276$ & 1,031 & 26,681 \\
\hline 5 & 245 & 241 & $180,915,260$ & 1,229 & 24,668 \\
\hline 6 & 213 & 232 & $171,115,067$ & 1,253 & 22,989 \\
\hline 7 & 344 & 323 & $159,138,663$ & 1,578 & 22,291 \\
\hline 8 & 377 & 378 & $146,364,022$ & 1,037 & 20,571 \\
\hline 9 & 283 & 209 & $141,213,431$ & 1,226 & 17,122 \\
\hline 10 & 374 & 324 & $135,534,747$ & 1,143 & 19,255 \\
\hline 11 & 277 & 230 & $135,006,516$ & 1,367 & 18,014 \\
\hline 12 & 283 & 236 & $133,851,895$ & 1,221 & 19,079 \\
\hline 13 & 157 & 152 & $115,169,878$ & 605 & 13,980 \\
\hline 14 & 184 & 123 & $107,349,540$ & 788 & 12,252 \\
\hline 15 & 122 & 121 & $102,531,392$ & 792 & 10,987 \\
\hline 16 & 415 & 287 & $90,354,753$ & 1,491 & 13,269 \\
\hline 17 & 322 & 224 & $81,195,210$ & 1,634 & 11,454 \\
\hline 18 & 140 & 158 & $78,077,248$ & 507 & 10,875 \\
\hline 19 & 351 & 180 & $59,128,983$ & 2,541 & 9,706 \\
\hline 20 & 263 & 205 & $63,025,520$ & 801 & 9,034 \\
\hline 21 & 150 & 139 & $48,129,895$ & 365 & 5,658 \\
\hline 22 & 256 & 195 & $51,304,566$ & 719 & 5,459 \\
\hline $\mathrm{X}$ & 25 & 21 & $155,270,560$ & 896 & 22,705 \\
\hline $\mathrm{Y}$ & 2 & 3 & $59,373,566$ & 181 & 5,493 \\
\hline
\end{tabular}

FTD, Trifluridine; BrdU, 5-bromo-2'-deoxyuridine. ${ }^{1}$ Annotations of hg19 human genome were downloaded via UCSC genome browser (https://genome.ucsc.edu/cite.html).

extracellular matrix structural components such as $A C A N$, $B G N, C H A D$, and $C O L 12 A 1$, while BrdU peaks were near genes involved in cysteine-type endopeptidases inhibitor activity such as ARRB1, BIRC7, LCN1, and CST1.

The significant motifs in FTD-ChIP fragments. The significant motifs found by the MEME program are shown in Figure 3. Significant motifs contained di-nucleotide repeat such as (GT)n, and tri-nucleotide repeat such as (GGT)n, which correlates with overlapping analysis results.

\section{Discussion}

This is the first study to apply ChIP-seq approach to assess DNA replication profile in FTD-treated human colorectal cancer cells to understand their cytotoxic mechanisms. DNA replication profile in FTD-treated cells showed that FTD was preferentially incorporated into genomic regions containing simple repeats, $\mathrm{CpG}$ islands, and gene bodies (exon and intron).

We previously reported that FTD is incorporated into HCT-116 genomic DNA during proliferation, and in a timedependent manner (7). In addition, we showed that FTD accumulates in cells while they are in the $\mathrm{G}_{2}$ phase. HCT116 cells treated with $5 \mu \mathrm{M}$ FTD for $8 \mathrm{~h}$ exhibited FTD incorporation with $4 \mathrm{pmol} / \mu \mathrm{g}$ DNA. To simplify the following calculation, assuming a normal diploid genome and random incorporation into genomic DNA, FTD molecule should be present at 2.7 FTD molecules per $1 \mathrm{kbp}$ length of DNA (1 FTD molecule per 351 bp length of DNA) in HCT116 cells exposed to $5 \mu \mathrm{M}$ FTD for $8 \mathrm{~h}$. According to such assumptions, ChIP-fragment might be detected equally throughout the genome. However, significant FTD ChIP peaks over input DNA were identified in this study, suggesting that FTD molecules were not randomly incorporated into the DNA. ChIP-peaks detected along genome from asynchronously growing cancer cells was thought to be reflecting the bias of DNA replication origins and the differences in sequence preference between FTD and BrdU during incorporation into DNA. One may assume that the incorporation of FTD predominantly into simple repeat regions is a random phenomenon. However, based on base contents, such simple probabilities could not explain why FTD active regions overlap with tetra-nucleotide repeats (20.6\% out of simple repeat) by 1116 times higher than with mononucleotide repeat $(6.5 \%$ out of simple repeat), while random control overlaps with tetra-nucleotide repeat by 3 times higher than with mono-nucleotide repeat, which was in agreement with simple probabilities. A thermodynamic interaction due to spatially large fluorine molecules in FTD 


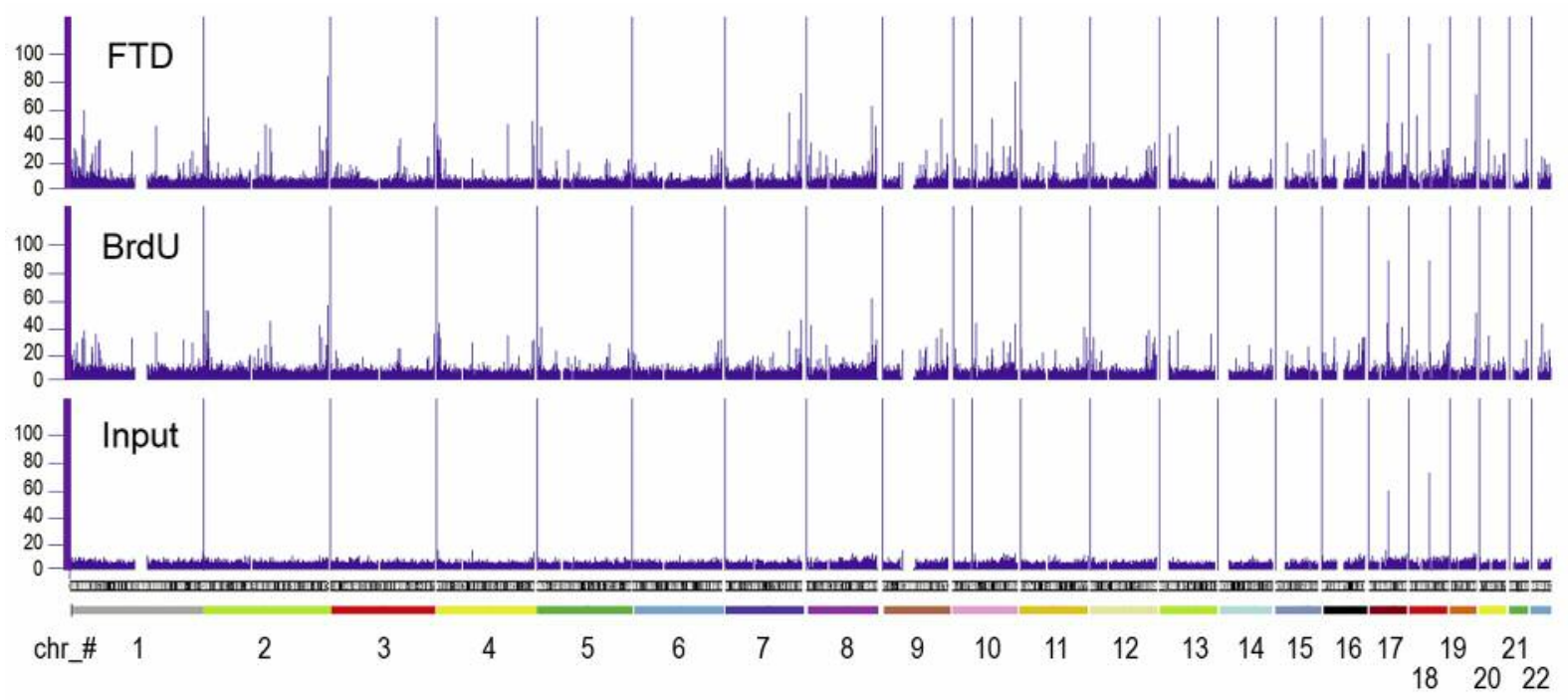

Figure 1. The ChIP fragment densities along the genome.

Table II. Overlapping percentage of active regions with genomic annotations.

\begin{tabular}{lcccc}
\hline Genome regions & \multicolumn{4}{c}{ Overlapping (\%) } \\
\cline { 2 - 5 } & $\begin{array}{c}\text { FTD } \\
\text { active } \\
\text { regions }\end{array}$ & $\begin{array}{c}\text { BrdU } \\
\text { active } \\
\text { regions }\end{array}$ & $\begin{array}{c}\text { Random } \\
\text { control } \\
\text { A }\end{array}$ & $\begin{array}{c}\text { Random } \\
\text { control } \\
\text { B }\end{array}$ \\
\hline CpG island & 2.7 & 2.6 & 1.2 & 1.0 \\
Replication origin* & 5.4 & 4.6 & 1.5 & 1.5 \\
Txn_Factor_ChIP & 69.2 & 68.8 & 41.0 & 40.7 \\
Simple repeat & $77.6^{* *}$ & 72.8 & 6.5 & 6.6 \\
microsatellite & 0.6 & 0.6 & 0.6 & 0.7 \\
Segmental duplication & 5.0 & 5.3 & 5.4 & 5.2 \\
genes and gene predictions & $49.4^{* *}$ & 46.8 & 43.4 & 43.7 \\
coding exons & $4.5^{* *}$ & 3.5 & 3.8 & 4.0 \\
5'UTR-exons & 1.8 & 1.4 & 1.9 & 1.9 \\
3'UTR-exons & 1.8 & 1.6 & 2.2 & 2.2 \\
intron & 48.1 & 45.7 & 42.3 & 42.3 \\
\hline
\end{tabular}

FTD, Trifluridine; BrdU, 5-bromo- 2 '-deoxyuridine. *Annotation of replication origin based on ini-seq BED files (20). **Significant difference between FTD and BrdU by the Fisher's exact test $(p<0.05)$.

incorporated DNA may explain these discordances of FTD incorporation into mononucleotide repeat region. Koseki $e t$ $a l$. reported that incorporation of FTD into DNA destabilizes the conformation of double-helical DNA via halogen bonding and dispersion interactions (16). Moreover, FTD also suppresses dTTP biosynthesis, which enhances FTD incorporation into DNA and thus its cytotoxicity (6). In line with this evidence, Anglana et al. reported that nucleotide pool modulates DNA replication origin choice and inter-
Table III. Overlapping percentage of active regions with simple repeats.

\begin{tabular}{lcccc}
\hline & \multicolumn{4}{c}{ Overlapping (\%) } \\
\cline { 2 - 5 } Simple repeats & $\begin{array}{c}\text { FTD } \\
\text { active } \\
\text { regions }\end{array}$ & $\begin{array}{c}\text { BrdU } \\
\text { active } \\
\text { regions }\end{array}$ & $\begin{array}{c}\text { Random } \\
\text { control } \\
\text { A }\end{array}$ & $\begin{array}{c}\text { Random } \\
\text { control } \\
\text { B }\end{array}$ \\
\hline Mononucleotide_repeat & 0.02 & 0.02 & 0.47 & 0.48 \\
Di-nucleotide_repeat & 45.2 & 47.3 & 3.1 & 3.2 \\
Tri-nucleotide_repeat & 9.1 & 10.3 & 0.73 & 0.68 \\
Tetra-nucleotide_repeat & 22.3 & 16.3 & 1.4 & 1.5 \\
Penta-nucleotide_repeat & 4.2 & 3.7 & 0.74 & 0.66 \\
Hexa-nucleotide_repeat & 7.2 & 7.7 & 0.42 & 0.44 \\
\hline
\end{tabular}

FTD, Trifluridine; BrdU, 5-bromo-2'-deoxyuridine.

origin spacing (17). The change in nucleotide pool caused by FTD exposure may accentuate DNA replication origin, and lead to the differences in ChIP profiles between FTD and BrdU. We have extracted several genes potentially associated with FTD-incorporated regions using the GREAT algorithm; however, a transcriptomic analysis is necessary in future studies to check whether there are specific FTD-responsive genes. The influence of FTD incorporation on the replication stress of DNA strand should be taken in account as well (18).

Limitations of the present study were the coarse-resolution in ChIP-seq analysis and insufficient knowledge of untranslated regions in the human genome. The ChIP-seq analysis is suitable to show the landscape of FTD molecule incorporation throughout the entire genome, but its analytical resolution is not sufficient. The detection of FTD molecules 


\section{A}

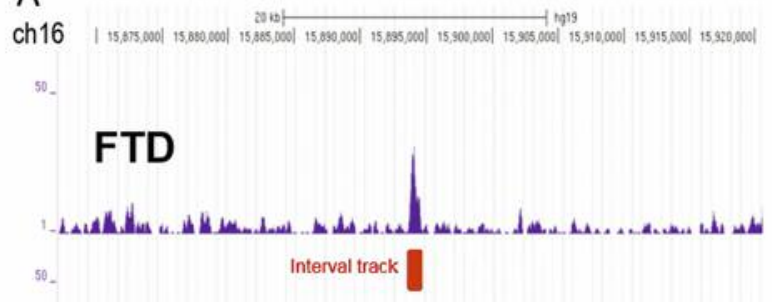

\section{BrdU}

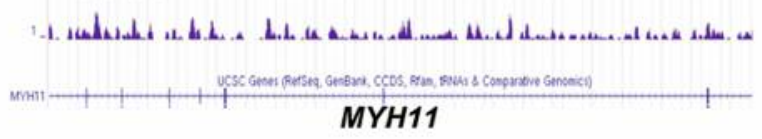

B

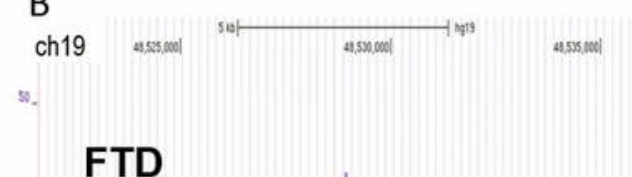

FTD

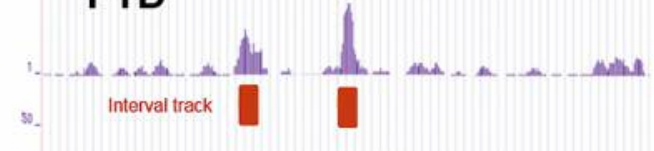

\section{BrdU}

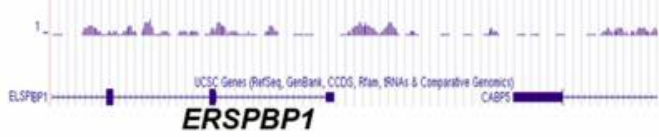

C

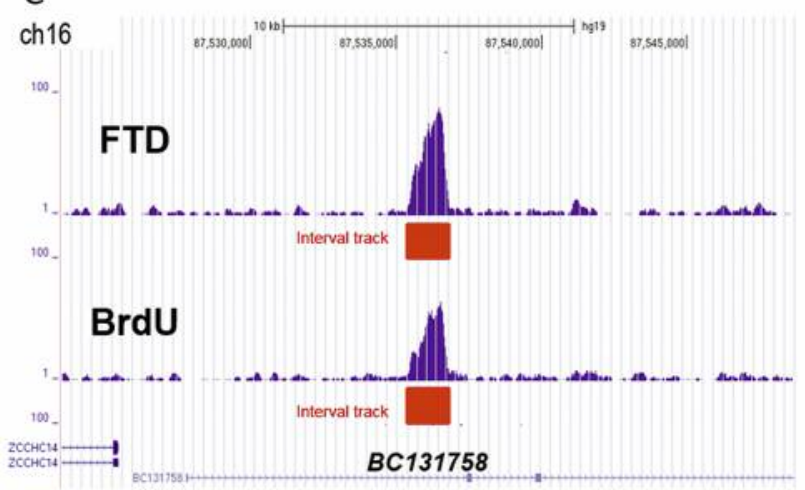

Figure 2. The examples of FTD specific ChIP peaks $(A, B)$ and common ChIP peaks $(C)$. Location and values of ChIP peaks were visualized in UCSC genome browser (http://genome.ucsc.deu/index.html).

with a single base resolution may be achieved by a new technique, such as tunnel-current based single-molecule electrical sequencing using nanopore integrated nanogap devices, or single molecule, real-time sequencing technology (19). Progress in the biology of untranslated regions of the human genome might be important to uncover the underlying molecular mechanisms leading cancer cells to death caused

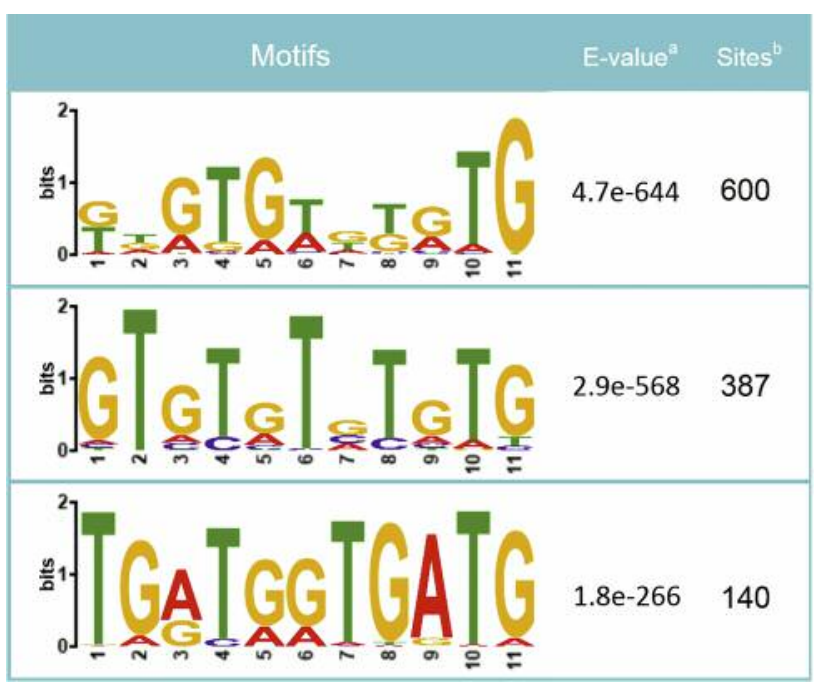

Figure 3. The significant motifs identified from ChIP-seq. ${ }^{\text {a The E-value }}$ is an estimate of the expected number of motifs with the given log likelihood ratio, and with the same width and site count, that one would find in a similarly sized set of random sequences. ${ }^{b}$ The number of sites contributing to the construction of the motif.

by FTD as well. A more precise analysis of FTD incorporation into DNA may lead to the development of a new treatment strategy for cancer.

\section{Conflicts of Interest}

There are no conflicts of interest to declare.

\section{Authors' Contributions}

T. Kobunai and K. Matsuoka conceived and designed the experiments, and acquired the data. T. Kobunai analyzed and interpreted the data (e.g., statistical analysis, biostatistics, computational analysis). Writing, review, and/or revision of the manuscript were performed by T. Kobunai, K. Matsuoka, and T. Takechi. T. Kobunai and T. Takechi supervised the study.

\section{Acknowledgements}

This research did not receive any specific grant from funding agencies in the public, commercial, or not-for-profit sectors.

\section{References}

1 Mayer RJ, Cutsem EV, Falcone A, Yoshino T, Carbonero RG, Mizunuma N, Yamazaki K, Shimada Y, Tabernero J, Komatsu Y, Sobrero A, Boucher E, Peeters M, Tran B, Lenz HH, Zaniboni A, Hochster H, Cleary JM, Prenen H, Benedetti F, Mizuguchi H, Makris L, Ito M, Ohtsu A and RECOURSE Study Group: Randomized trial of TAS-102 for refractory metastatic colorectal cancer. N Engl J Med 372: 1909-1919, 2015. PMID: 25970050. DOI: 10.1056/NEJMoa1414325 
2 Kuboki Y, Nishina T, Shinozaki E, Yamazaki K, Shitara K, Okamoto W, Kajiwara T, Matsumoto T, Tsushima T, Mochizuki N, Nomura S, Doi T, Sato A, Ohtsu A and Yoshino T: TAS-102 plus bevacizumab for patients with metastatic colorectal cancer refractory to standard therapies (C-TASK FORCE): an investigator-initiated, open-label, single-arm, multicentre, phase 1/2 study. Lancet Oncol 18(9): 1172-1181, 2017. PMID: 28760399. DOI: $10.1016 /$ S1470-2045(17)30425-4

3 Heidelberger C, Parsons DG and Remy DC: Syntheses of 5trifluoromethyluracil and 5-trifluoromethyl-2'-deoxyuridine. J Med Chem 7: 1-5, 1964. PMID: 14186018.

4 Emura T, Nakagawa F, Fujioka A, Ohshimo H, Yokogawa T, Okabe $\mathrm{H}$ and Kitazato $\mathrm{K}$ : An optimal dosing schedule for a novel combination antimetabolite, TAS-102, based on its intracellular metabolism and its incorporation into DNA. Int J Mol Med 13: 249-255, 2004. PMID: 14719131.

5 Temmink OH, Bijnsdorp IV, Prins HJ, Losekoot N, Adema AD, Smid K, Honeywell RJ, Ylstra B, Eijk PP, Fukushima M and Peters GJ: Trifluorothymidine resistance is associated with decreased thymidine kinase and equilibrative nucleoside transporter expression or increased secretory phospholipase A2. Mol Cancer Ther 9: 1047-1057, 2010. PMID: 20371715. DOI: 10.1158/1535-7163.MCT-09-0932

6 Tanaka N, Sakamoto K, Okabe H, Fujioka A, Yamamura K, Nakagawa F, Nagase $H$, Yokogawa $T$, Oguchi $K$, Ishida $K$, Osada A, Kazuno H, Yamada Y and Matsuo K: Repeated oral dosing of TAS-102 confers high trifluridine incorporation into DNA and sustained antitumor activity in mouse models. Oncol Rep 32: 2319-2326, 2014. PMID: 25230742. DOI: 10.3892/ or.2014.3487

7 Matsuoka $\mathrm{K}$, Iimori $\mathrm{M}$, Niimi $\mathrm{S}$, Tsukihara $\mathrm{H}$, Watanabe $\mathrm{S}$, Kiyonari S, Kiniwa M, Ando K, Tokunaga E, Saeki H, Oki E, Maehara $\mathrm{Y}$ and Kitao $\mathrm{H}$ : Trifluridine induces p53-dependent sustained $G_{2}$ phase arrest with its massive misincorporation into DNA and few DNA strand breaks. Mol Cancer Ther 14: 10041013, 2015. PMID: 25700705. DOI: 10.1158/1535-7163.MCT14-0236

8 Sakamoto K, Yokogawa T, Ueno H, Oguchi K, Kazuno H, Ishida K, Tanaka N, Osada A, Yamada Y, Okabe H and Matsuo K: Crucial roles of thymidine kinase 1 and deoxy UTPase in incorporating the antineoplastic nucleosides trifluridine and 2 'deoxy-5-fluorouridine into DNA. Int J Oncol 46(6): 2327-34, 2015. PMID: 25901475. DOI: 10.3892/ijo.2015.2974

9 Leonhardt H, Page AW, Weier HU and Bestor TH: A targeting sequence directs DNA methyltransferase to sites of DNA replication in mammalian nuclei. Cell 71(5): 865-873, 1992. PMID: 1423634.

10 Michishita E, Matsumura N, Kurahashi T, Suzuki T, Ogino H, Fujii $\mathrm{M}$ and Ayusawa D: 5-Halogenated thymidine analogues induce a senescence-like phenomenon in HeLa cells. Biosci Biotechnol Biochem 66: 877-879, 2002. PMID: 12036067. DOI: $10.1271 / \mathrm{bbb} .66 .877$
11 Kitao H, Morodomi Y, Niimi S, Kiniwa M, Shigeno K, Matsuoka K, Kataoka Y, Iimori M, Tokunaga E, Saeki H, Oki E and Maehara Y: The antibodies against 5-bromo-2'-deoxyuridine specifically recognize trifluridine incorporated into DNA. Sci Rep 6: 25286, 2016. PMID: 27137226. DOI: 10.1038/srep25286

12 Egan B, Yuan CC, Craske ML, Labhart P, Guler GD, Arnott D, Maile TM, Busby J, Henry C, Kelly TK, Tindell CA, Jhunjhunwala S, Zhao F, Hatton C, Bryant BM, Classon M and Trojer P: An alternative approach to ChIP-Seq normalization enables detection of genome-wide changes in histone $\mathrm{H} 3$ lysine 27 trimethylation upon EZH2 inhibition. PLoS One 11(11): e0166438, 2016. PMID: 27875550. DOI: 10.1371/journal. pone. 0166438

$13 \mathrm{Li} \mathrm{H}$ and Durbin R: Fast and accurate short read alignment with Burrows-Wheeler transform. Bioinformatics 25(14): 1754-1760, 2009. PMID: 19451168. DOI: 10.1093/bioinformatics/btp324

14 Bailey TL and Elkan C: Fitting a mixture model by expectation maximization to discover motifs in biopolymers. Proceedings of the Second International Conference on Intelligent Systems for Molecular Biology, pp. 28-36, AAAI Press, Menlo Park, California, 1994.

15 McLean CY, Bristor D, Hiller M, Clarke SL, Schaar BT, Lowe $\mathrm{CB}$, Wenger $\mathrm{AM}$ and Bejerano G: GREAT improves functional interpretation of cis-regulatory regions. Nat Biotechnol 28: 495501, 2010. PMID: 20436461. DOI: 10.1038/nbt.1630

16 Koseki J, Tsunekuni K, Konno M, Nishida N, Kawamoto K, Doki $\mathrm{Y}$, Mori M and Ishii H: Thermodynamic and molecular orbital analysis of the effects caused by incorporation of novel anti-tumor agent Trifluridine to DNA. Abstract 2706: AACR 107th Annual Meeting, 2016. DOI: 10.1158/1538-7445.AM2016 -2706

17 Anglana M, Apiou F, Bensimon A and Debatisse M: Dynamics of DNA replication in mammalian somatic cells: nucleotide pool modulates origin choice and interorigin spacing. Cell 114(3): 385-394, 2008. PMID: 12914702.

18 Kitao H, Iimori M, Kataoka Y, Wakasa T, Tokunaga E, Saeki H, Oki E and Maehara Y: DNA replication stress and cancer chemotherapy. Cancer Sci 109(2): 264-271, 2018. PMID: 29168596. DOI: $10.1111 /$ cas.13455

19 Maitra RD, Kim J and Dunbar WB: Recent advances in nanopore sequencing. Electrophoresis 33(23): 3418-3428, 2012. PMID: 23138639. DOI: 10.1002/elps.201200272

20 Langley AR, Gräf S, Smith JC and Krude T: Genome-wide identification and characterisation of human DNA replication origins by initiation site sequencing (ini-seq). Nucleic Acids Res 44(21): 10230-10247, 2016. PMID: 27587586. DOI: 10.1093/ nar/gkw760
Received February 18, 2019

Revised March 23, 2019

Accepted March 26, 2019 